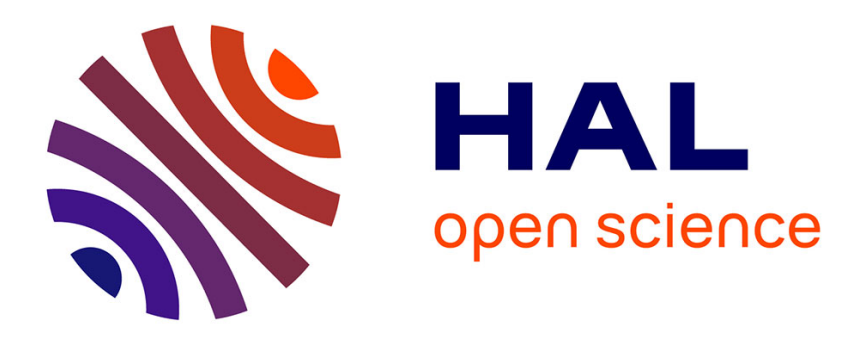

\title{
Stability analysis for sampled-data systems with a time-varying period
}

Alexandre Seuret

\section{To cite this version:}

Alexandre Seuret. Stability analysis for sampled-data systems with a time-varying period. CDC 2009

- 48th IEEE Conference on Decision and Control, Dec 2009, Shanghai, China. pp.6. hal-00403252v2

\section{HAL Id: hal-00403252 \\ https://hal.science/hal-00403252v2}

Submitted on 21 Sep 2009

HAL is a multi-disciplinary open access archive for the deposit and dissemination of scientific research documents, whether they are published or not. The documents may come from teaching and research institutions in France or abroad, or from public or private research centers.
L'archive ouverte pluridisciplinaire HAL, est destinée au dépôt et à la diffusion de documents scientifiques de niveau recherche, publiés ou non, émanant des établissements d'enseignement et de recherche français ou étrangers, des laboratoires publics ou privés. 


\title{
Stability analysis for sampled-data systems with a time-varying period
}

\author{
Alexandre Seuret \\ alexandre.seuret@gipsa-lab.inpg.fr \\ NeCS Team, Department of Automatic Control, \\ GIPSA-Lab-CNRS UMR 5216, Grenoble, France
}

\begin{abstract}
This paper proposes a novel stability analysis of linear systems with sampled-data inputs. Inspired by the inputdelay approach and the stability of impulsive systems, this method provides novel sufficient stability conditions. The stability analysis concerns both constant and time-varying sampling periods. The delay-dependent conditions are expressed using computable linear matrix inequalities. Several examples show the efficiency and the limitation of such stability criteria.

Index Terms-Sampled data controller, Time-delay, delaydependent stability, Lyapunov-Krasovskii functionals (LKF), linear matrix inequality (LMI)
\end{abstract}

\section{INTRODUCTION}

In the last decades, a large attention has been taken to Networked Control Systems (NCS) (see [5], or [15]). Such systems are controlled systems containing several distributed plants which are connected through a communication network. In such applications, a heavy temporary load of computation in a processor can corrupt the sampling period of a certain controller. On the other side, the sampling period can be scheduled in the design in order to avoid this load. In both cases, the variations of the sampling period will affect the stability properties. It is thus an important issue to develop robust stability conditions with respect to the variations of sampling period.

Sampled-data systems have already been studied in the literature [2], [16], [17] and the references therein. It is now reasonable to design controllers which guarantee the robustness of the solutions of the closed-loop system under periodic samplings. However the case of asynchronous samplings still leads to several open problems such that the guarantee of stability whatever the sampling period lying in an interval. Recently, several articles drive the problem of time-varying periods based on a discrete-time approach, [4], [13]. Note that discrete-time approaches do not fit with the case of uncertain systems or systems with timevarying parameters. Recent papers considered the modelling of continuous-time systems with sampled-data control in the form of continuous-time systems with delayed control input. In [3], a Lyapunov-Krasovskii approach is introduced. Improvements are provided in [9], using the small gain theorem and in [10] based on the analysis of impulsive systems. These appraoches are very relevant because it is easy to consider time-varying sampling periods and they can

This research is supported by FeedNetBack project, FP7- ICT-2007-2: http://www.feednetback.eu/. deal with uncertain systems (see [3] and [10]). Nevertheless, these sufficient conditions are still conservative. This means that the sufficient conditions obtained by continuous time approaches are not able to guarantee asymptotic stability whereas the systems is stable.

The article proposes novel sufficient stability conditions to ensure stability of linear time-varying systems. Those conditions are based on the continuous-time approach and the stability of impulsive systems. They provide a larger upperbound of the allowable sampling period than the existing ones (based on the continuous time approach). In this paper, asymptotic and exponential stability are considered.

This article is organized as follows. The next section formulates the problem. Section III and IV respectively deals with the analysis of asymptotic and exponential stability. Some examples and simulations are provided and show the efficiency of the method in Section V.

Notations. Throughout the article, for a n-dimensional state vector $x$ and a non-negative delay $\tau, x_{t}$ denotes a function such that $x_{t}(\theta)=x(t-\theta)$ for all $\theta \in[-\tau, 0]$. The superscript ' $T$ ' stands for the matrix transposition. The notation $P>0$ for $P \in \mathbb{R}^{n \times n}$ means that $P$ is a symmetric and positive definite matrix. The symbols $I$ and 0 represent the identity and the zero matrices of appropriate dimension.

\section{PRoblem FORMUlation}

Consider the linear system with a sampled-data input:

$$
\dot{x}(t)=A x(t)+B u\left(t_{k}\right)
$$

where $x \in \mathbb{R}^{n}$ and $u \in \mathbb{R}^{m}$ represent the state variable and the input vector. The matrices $A$ and $B$ are constant and of appropriate dimension. We are looking for a piecewiseconstant control law of the form $u(t)=u_{d}\left(t_{k}\right), t_{k} \leq t<$ $t_{k+1}$, where $u_{d}$ is a discrete-time control signal and $0=t_{0}<$ $t_{1}<\ldots<t_{k}<\ldots$ are the sampling instants. Our objective is to ensure the stability of the system together with a statefeedback controller of the form:

$$
u(t)=K x\left(t_{k}\right), t_{k} \leq t<t_{k+1} .
$$

where the gain $K$ in $\mathbb{R}^{n \times m}$ is given. Assume that the difference between two successive sampling instants satisfies

$$
0<t_{k+1}-t_{k} \leq \tau_{m} \forall k \geq 0 .
$$

Several authors investigated in guaranteing the stability of such a system. In [3], a first approach was introduced. It allows assimilating sampling effects as the ones of a 

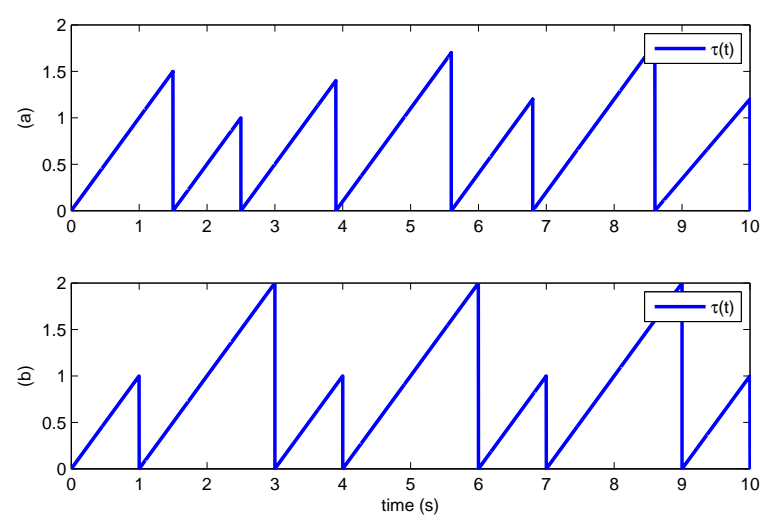

Fig. 1. Examples of two sampling delays with time-varying period: (a) shows the case of a of sampling periods which vary arbitrarily, (b) represents the case of a repetitive sequence of two known and exact samplings.

particular delay. Substituting (2) into (1), we obtain the following closed-loop system:

$$
\begin{aligned}
& \dot{x}(t)=A x(t)+A_{d} x(t-\tau(t)), \\
& \tau(t)=t-t_{k}, t_{k} \leq t<t_{k+1} .
\end{aligned}
$$

where $A_{d}=B K$. Example of time-varying delay, $\tau(t)$, representing a sampling with a time-varying period is presented in Figure 1. In the case of known and exact sampling, or periodic scheduling (b) in Figure 1, the reader can refer for instance to [7] for a stability analysis. However for case (a), this method is no more applicable since the sampling period is not known.

From (3), it follows that $\tau(t) \leq \tau_{m}$ since $\tau(t) \leq t_{k+1}-t_{k}$. We will further consider (4) as a linear system with uncertain and bounded delay. The sufficient stability conditions from [3] were designed to deal with all kind of delay functions. As sampled-data systems are systems subject to a particular delay, this approach was conservative with respect to the maximum allowable sampling period. In [10], the authors introduce a new type of Lyapunov-Krasovskii functionals which depends explicitly and linearly on the delay function. This allows reducing the conservatism of the results. In this article, a new type of Lyapunov-Krasovskii functional is introduced to especially consider sampled delays. This method leads to less conservative result.

\section{ASYMPTOTIC STABILITY ANALYSIS}

\section{A. Time-varying sampling period}

Consider system (4) with a time-varying sampling period satisfying (3). The following theorem holds:

Theorem 1: Assume that there exist symmetric positive definite matrices $P, R$ and $S \in \mathbb{R}^{n \times n}$ and a matrix $N \in$ $\mathbb{R}^{2 n \times n}$ that satisfy:

$$
\begin{gathered}
\Pi_{1}+\tau_{m} \Pi_{2}<0, \\
{\left[\begin{array}{cc}
\Pi_{1} & \tau_{m} N \\
* & -\tau_{m} R
\end{array}\right]<0,}
\end{gathered}
$$

where

$$
\begin{aligned}
\Pi_{1}= & M_{1}^{T} P M_{3}+M_{3}^{T} P M_{1}-M_{2}^{T} S M_{2} \\
& -N M_{2}-M_{2}^{T} N^{T}, \\
\Pi_{2}= & M_{2}^{T} S M_{3}+M_{3}^{T} S M_{2}+M_{3}^{T} R M_{3},
\end{aligned}
$$

and the matrices $M_{i}$, for $i=1,2,3$ are given by:

$$
\begin{gathered}
M_{1}=\left[\begin{array}{ll}
I & 0
\end{array}\right], M_{2}=\left[\begin{array}{ll}
I & -I
\end{array}\right], \\
M_{3}=\left[\begin{array}{ll}
A & A_{d}
\end{array}\right] .
\end{gathered}
$$

Then system (4) is asymptotically for any time-varying period less than $\tau_{m}$.

Proof: Inspired from the technic introduced in [10], consider the following form of functional:

$$
\begin{aligned}
V\left(t, x_{t}\right) & =x^{T}(t) P x(t)+\left(\tau_{m}-\tau(t)\right) \zeta_{0}^{T}(t) S \zeta_{0}(t) \\
& +\left(\tau_{m}-\tau(t)\right) \int_{t_{k}}^{t} \dot{x}^{T}(s) R \dot{x}(s) d s
\end{aligned}
$$

where

$$
\zeta_{0}(t)=x(t)-x\left(t_{k}\right) .
$$

Consider the functional $V$ just before the sampling instant $t_{k}$, denoted as $t_{k}^{-}$. The Lyapunov-Krasovskii functionals $V\left(t_{k}^{-}, x_{t_{k}^{-}}\right)$is strictly greater than $x\left(t_{k}\right)^{T} P x\left(t_{k}\right)$. Just after the sampling instant, i.e. $t=t_{k}^{+}$, the two last terms of the functional are zero, because $\tau\left(t_{k}^{+}\right)=0$ ). Then it means that the functional is decreasing discontinuously at each sampled instant (see [10] for more details). Thus one has:

$$
V\left(t_{k}^{-}, x_{t_{k}^{-}}\right)>V\left(t_{k}^{+}, x_{t_{k}^{+}}\right)=x\left(t_{k}\right)^{T} P x\left(t_{k}\right) .
$$

To prove the stability of the system, one has to ensure that $V$ is decreasing within each period. An expression of the derivative of $V$ during one sampling period is derived:

$$
\begin{aligned}
\dot{V}\left(t, x_{t}\right)= & 2 x^{T}(t) P \dot{x}(t)+2\left(\tau_{m}-\tau(t)\right) \zeta_{0}^{T}(t) S \dot{x}(t) \\
& +\left(\tau_{m}-\tau(t)\right) \dot{x}^{T}(t) R \dot{x}(t)-\zeta_{0}^{T}(t) S \zeta_{0}(t) \\
& -\int_{t_{k}}^{t} \dot{x}^{T}(s) R \dot{x}(s) d s .
\end{aligned}
$$

The next step of the proof consists in rewriting the expression of $\dot{V}$ using the vector $\xi(t)=\left[\begin{array}{ll}x^{T}(t) & x^{T}\left(t_{k}\right)\end{array}\right]^{T}$. It is easy to see that:

$$
\begin{aligned}
& x(t)=M_{1} \xi(t), \quad \zeta_{0}=x(t)-x\left(t_{k}\right)=M_{2} \xi(t), \\
& \dot{x}(t)=A x(t)+A_{d} x\left(t_{k}\right)=M_{3} \xi(t) .
\end{aligned}
$$

This leads to:

$$
\begin{aligned}
& \dot{V}\left(t, x_{t}\right)=\xi^{T}(t)\left[2 M_{1}^{T} P M_{3}-M_{2}^{T} S M_{2}\right. \\
& \left.\quad+\left(\tau_{m}-\tau(t)\right)\left(2 M_{3}^{T} S M_{2}+M_{3}^{T} R M_{3}\right)\right] \xi(t) \\
& \quad-\int_{t_{k}}^{t} \dot{x}^{T}(s) R \dot{x}(s) d s .
\end{aligned}
$$

Noting that $\zeta_{0}(t)-\int_{t_{k}}^{t} \dot{x}(s) d s=0$, the term $2 \xi^{T}(t) N M_{2} \xi(t)-2 \xi^{T}(t) N \int_{t_{k}}^{t} \dot{x}(s) d s=0$ is added to the previous equation. Noting that $R$ is positive definite, a classical bounding ensures that:

$$
2 \xi^{T}(t) N \dot{x}(s) \leq \xi^{T}(t) N R^{-1} N^{T} \xi(t)+\dot{x}^{T}(s) R \dot{x}(s) d s .
$$

Integrating the previous inequality in the interval $\left[t_{k}, t\right]$ where $\dot{x}$ is continuous, the following inequality is obtained:

$$
\begin{aligned}
-2 \xi^{T}(t) N M_{2} \xi(t)+\tau(t) & \xi^{T}(t) N R^{-1} N^{T} \xi(t) \\
& +\int_{t_{k}}^{t} \dot{x}^{T}(s) R \dot{x}(s) d s \geq 0 .
\end{aligned}
$$


Then adding the previous inequality to (8), the following inequality is obtained for all $t \in\left[t_{k}, t_{k+1}[\right.$ :

$$
\begin{aligned}
\dot{V}\left(t, x_{t}\right) \leq & \xi^{T}(t)\left[\Pi_{1}+\tau_{m} \Pi_{2}\right. \\
& \left.+\tau(t)\left(N R^{-1} N^{T}-\Pi_{2}\right)\right] \xi(t) .
\end{aligned}
$$

The objective is to ensure that $\dot{V}$ is definite negative for all values of the delay $\tau(t) \in\left[0, \tau_{m}[\right.$. As this equation depends linearly on the delay function, it is necessary and sufficient to ensure the negativity of the matrix for $\tau(t)=0$ and $\tau_{m}$ to prove that $\dot{V}$ is definite negative within the interval $\left[t_{k}, t_{k+1}\right]$. Applying the Schur complement on the vertice $\tau(t)=\tau_{m}$ leads to the stability conditions of Theorem 1 .

Thus combining the facts that $V$ is decreasing within all intervals $\left[t_{k}, t_{k+1}\right.$ [ and that (7) holds, we prove that $V$ converges asymptotically to 0 . Consequently the solutions of (4) are asymptotically stable.

The sufficient stability conditions of Theorem 1 are very similar to the ones from [10]. The only difference comes from the integral term with the matrix $R$. In [10], the employed functional is of the following form:

$$
\begin{aligned}
V^{\prime}\left(t, x_{t}\right) & =x^{T}(t) P x(t)+\left(\tau_{m}-\tau(t)\right) \zeta_{0}^{T}(t) S \zeta_{0}(t) \\
& +\int_{t_{k}}^{t}\left(\tau_{m}-t+s\right) \dot{x}^{T}(s) R \dot{x}(s) d s .
\end{aligned}
$$

To understand the difference between this functional and the one used in Theorem 1 , one can see that for all $t \in$ $\left[t_{k}, t_{k+1}[:\right.$

$$
V^{\prime}\left(t, x_{t}\right)=V\left(t, x_{t}\right)+\int_{t_{k}}^{t} \tau(s) \dot{x}^{T}(s) R \dot{x}(s) d s
$$

Consequently, one has:

$$
\dot{V}^{\prime}\left(x_{t}\right)=\dot{V}\left(x_{t}\right)+\tau(t) \dot{x}^{T}(s) R \dot{x}(s) d s,
$$

It is thus clear that $\dot{V}$ can be negative whereas $\dot{V}^{\prime}$ is positive. In the previous equation, the positive term $\tau(t) \dot{x}^{T}(t) R \dot{x}(t)$ makes the conditions more conservatism than the sufficient stability conditions from [10].

\section{B. Constant sampling period}

Following the line of [10], Theorem 1 can be improved in the case of constant sampling period. The following theorem is formulated:

Theorem 2: Assume that there exist symmetric positive definite matrices $P, R$ and $S \in \mathbb{R}^{n \times n}$ and two matrices $U \in \mathbb{R}^{n \times n}$ and $N \in \mathbb{R}^{2 n \times n}$ such that:

$$
\bar{\Pi}_{1}+\tau_{m} \bar{\Pi}_{2}<0, \quad\left[\begin{array}{cc}
\bar{\Pi}_{1} & \tau_{m} N \\
* & -\tau_{m} R
\end{array}\right]<0,
$$

where

$$
\begin{aligned}
& \bar{\Pi}_{1}=\Pi_{1}-M_{4}^{T} U M_{2}-M_{2}^{T} U^{T} M_{4}, \\
& \bar{\Pi}_{2}=\Pi_{2}+M_{4}^{T} U M_{3}+M_{3}^{T} U^{T} M_{4},
\end{aligned}
$$

and where $M_{i}=1,2,3$ are given in Theorem 1 and where $M_{4}=\left[\begin{array}{ll}0 & I\end{array}\right]$. Then system (4) is asymptotically stable for the constant sampling period $\tau_{m}$.

Proof: The proof follows the line of Theorem 1. Consider the functional:

$$
V_{1}\left(t, x_{t}\right)=V\left(t, x_{t}\right)+V_{2}\left(t, x_{t}\right),
$$

where $V_{2}\left(t, x_{t}\right)=2\left(\tau_{m}-\tau(t)\right) x^{T}\left(t_{k}\right) U \zeta_{0}(t)$. Note that $V_{2}\left(t, x_{t}\right)$ is not necessary positive. Thanks to the constant and known sampling period, one has:

$$
\forall k>0, \quad V_{2}\left(t_{k}^{-}, x_{t_{k}^{-}}\right)=V_{2}\left(t_{k}^{+}, x_{t_{k}^{+}}\right)=0 .
$$

It is thus clear that $V_{2}\left(t, x_{t}\right)$ is continuous over $\mathbb{R}$. This is the reason why this term can only be considered in the particular the case of constant sampling period. The differentiation of $V_{1}$ along the trajectories of (4) leads to:

$$
\begin{aligned}
\dot{V}_{1}\left(t, x_{t}\right)= & \dot{V}\left(t, x_{t}\right)+2\left(\tau_{m}-\tau(t)\right) x^{T}\left(t_{k}\right) U \dot{x}(t) \\
& -2 x^{T}\left(t_{k}\right) U \zeta_{0}(t) .
\end{aligned}
$$

Noting that $x\left(t_{k}\right)=\left(M_{1}-M_{2}\right) \xi(t)$, the derivative of $V_{1}$ satisfies:

$$
\begin{aligned}
\dot{V}_{1}\left(t, x_{t}\right)= & \xi^{T}(t)\left[\bar{\Pi}_{1}+\tau_{m} \bar{\Pi}_{2}\right. \\
& \left.+\tau(t)\left(N R^{-1} N^{T}-\bar{\Pi}_{2}\right)\right] \xi(t) .
\end{aligned}
$$

Applying the same method as in Theorem 1, system (4) is asymptotically stable for the constant sampling periods, $\tau_{m}$, that satisfy (9).

\section{System with polytopic type uncertainties}

An extension to the case of systems with parameter uncertainties can be dealt by considering system (4) and with $A$ and $A_{d}$ from the uncertain polytope given by:

$$
\forall t \in \mathbb{R}^{+}, \quad \Omega(t)=\sum_{k=1}^{M} \lambda_{i}(t) \Omega_{i}
$$

where $\forall t \in \mathbb{R}^{+}$,

$$
\begin{array}{r}
\sum_{i=1}^{M} \lambda_{i}(t)=1 \\
\forall i=1, . ., M, \quad 0 \leq \lambda_{i}(t) .
\end{array}
$$

The $\Omega$ vertices of the polytope are described by $\Omega_{i}=$ $\left[A(i) \quad A_{d}(i)\right]$. The conditions of Theorems 1 and 2 are non linear with respect matrices $A$ and $A_{d}$ because of the term $M_{3}^{T} R M_{3}$. Thus a direct extension to the case of polytopic systems is not straightforward and requires some attention. They can be easily adapted as shown in the sequel:

Theorem 3: Assume that there exist symmetric positive definite matrices $P, R$ and $S \in \mathbb{R}^{n \times n}$ and a matrix $N \in$ $\mathbb{R}^{2 n \times n}$ such that:

$$
\begin{gathered}
{\left[\begin{array}{cc}
\Pi_{1}^{i}+\tau_{m} \Pi_{2}^{i} & \tau_{m} M_{3}^{i} R \\
* & -\tau_{m} R
\end{array}\right]<0} \\
{\left[\begin{array}{cc}
\Pi_{1}^{i} & \tau_{m} N \\
* & -\tau_{m} R
\end{array}\right]<0}
\end{gathered}
$$

where $M_{i}$, for $i=1,2$ are given in Theorem 1 and

$$
\begin{aligned}
\Pi_{1}^{i}= & M_{1}^{T} P M_{3}^{i}+M_{3}^{i} P M_{1}-M_{2}^{T} S M_{2} \\
& -N M_{2}-M_{2}^{T} N^{T}, \\
\Pi_{2}^{i}= & M_{2}^{T} S M_{3}^{i}+M_{3}^{i^{T}} S M_{2},
\end{aligned}
$$

and $M_{3}^{i}=\left[\begin{array}{ll}A^{i} & A_{d}^{i}\end{array}\right]$. System (4) with polytopic uncertainties is thus asymptotically stable for all sampling periods less than $\tau_{m}$.

Proof: Consider the first condition of Theorem 1. This inequality is not linear with respect to the matrix $A$ 
and $A_{d}$ because of the term $M_{3}^{T} R M_{3}$. Noting that it can be rewritten as $\left(M_{3}^{T} R\right) R^{-1}\left(R M_{3}\right)$, the Schur complement allows obtaining the first condition Theorem 3. Consider now the second LMI of Theorem 1 is linear with respect to the system parameters $A$ and $A_{d}$. Then extension to polytopic systems is straightforward. As both conditions become linear with respect to the matrices $A$ and $A_{d}$, one has to solve simultaneously the LMIs for all the $\Omega$ vertices. Thus, if the sufficient conditions (10) are satisfied, thus the system is asymptotically stable.

Remark 1: Theorem 3 can be extended to the case of constant sampling period by introducing the same terms as in Theorem 2. This leads to the following conditions:

$$
\begin{gathered}
\bar{\Pi}_{1}^{i}=\Pi_{1}^{i}-\left(M_{1}-M_{2}\right)^{T} U M_{2}-M_{2}^{T} U^{T}\left(M_{1}-M_{2}\right), \\
\bar{\Pi}_{2}^{i}=\Pi_{2}^{i}+\left(M_{1}-M_{2}\right)^{T} U M_{3}-M_{3}^{T} U^{T}\left(M_{1}-M_{2}\right) . \\
\text { IV. EXPONENTIAL STABILITY OF SYSTEMS WITH } \\
\text { SAMPLED INPUTS }
\end{gathered}
$$

In this section, a study of the convergence rate of the solutions of sampled-data systems is provided. The objective is to ensure that the solutions are bounded by a decreasing exponential function and to estimate the exponential rate $\alpha$ of convergence. Consider the definition of exponential convergence dedicated to time-delay systems:

Definition 1: [11] For given $\alpha>0$ and $\beta>1$, the closedloop system (1) is said to be $\alpha$-stable, or 'exponentially stable with the rate $\alpha$ ', if its solution $x\left(t ; t_{0}, \phi\right)$ satisfies:

$$
\left|x\left(t, t_{0}, \phi\right)\right| \leq \beta|\phi| e^{-\alpha\left(t-t_{0}\right)} .
$$

The following theorem holds:

Theorem 4: For a given $\alpha>0$, assume that there exist symmetric positive definite matrices $P, R$ and $S \in \mathbb{R}^{n \times n}$ and a matrix $N \in \mathbb{R}^{2 n \times n}$ that satisfy:

$$
\begin{gathered}
\Pi_{1}^{\alpha}+\tau_{m} \Pi_{2}^{\alpha}<0 \\
{\left[\begin{array}{cc}
\Pi_{1}^{\alpha} & \tau_{m} N \\
* & -\tau_{m}\left(1+2 \alpha \tau_{m}\right) R
\end{array}\right]<0,}
\end{gathered}
$$

where

$$
\begin{aligned}
& \Pi_{1}^{\alpha}=\Pi_{1}+2 \alpha\left(M_{1}^{T} P M_{1}-M_{2}^{T} R M_{2}\right), \\
& \Pi_{2}^{\alpha}=\Pi_{2}+2 \alpha M_{2}^{T} S M_{2},
\end{aligned}
$$

and where the matrices $M_{i}$, for $i=1,2,3$ are given in Theorem 1. System (4) is thus exponentially stable with an exponential decay rate $\alpha$ for any time-varying period less than $\tau_{m}$.

Proof: Consider $V$ from (6) and :

$$
W^{\alpha}\left(t, x_{t}\right)=\dot{V}\left(t, x_{t}\right)+2 \alpha V\left(t, x_{t}\right) \text {. }
$$

Sufficient exponential stability can be derived using the following requirement:

$$
W^{\alpha}\left(t, x_{t}\right)<0
$$

where $\alpha$ is a positive scalar. The following equality is provided:

$$
\begin{aligned}
& W^{\alpha}\left(t, x_{t}\right)=\xi^{T}(t)\left[2 M_{1}^{T} P M_{3}-M_{2}^{T} S M_{2}+2 \alpha M_{1}^{T} P M_{1}\right. \\
& \quad+\tau_{m}\left(2 M_{3}^{T} S M_{2}+M_{3}^{T} R M_{3}+2 \alpha M_{2}^{T} S M_{2}\right) \\
& \left.\quad-\tau(t)\left(2 M_{3}^{T} S M_{2}+M_{3}^{T} R M_{3}+2 \alpha M_{2}^{T} S M_{2}\right)\right] \xi(t) \\
& \quad-\int_{t_{k}}^{t} \dot{x}^{T}(s)\left(1-2 \alpha\left(\tau_{m}-\tau(t)\right) R \dot{x}(s) d s .\right.
\end{aligned}
$$

Consider the integral term of the equation above. It is split into two terms. The first one is $-\left(1-2 \alpha \tau_{m}\right) \int_{t_{k}}^{t} \dot{x}^{T}(s) R \dot{x}(s) d s$. The second one is $-2 \alpha \tau(t) \int_{t k}^{t} \dot{x}^{T}(s) R \dot{x}(s) d s$. Applying the Jensen's inequality, this last term is bounded by:

$$
\begin{aligned}
-\tau(t) \int_{t_{k}}^{t} \dot{x}^{T}(s) R \dot{x}(s) d s & \leq-\int_{t_{k}}^{t} \dot{x}^{T}(s) d s R \int_{t_{k}}^{t} \dot{x}(s) d s \\
& \leq-\xi(t) M_{2}^{T} R M_{2} \xi(t) .
\end{aligned}
$$

Noting that

$$
2 \xi^{T}(t) N M_{2} \xi(t)-2 \xi^{T}(t) N \int_{t_{k}}^{t} \dot{x}(s) d s=0,
$$

and that a classical bounding ensures that the following inequality holds:

$$
\begin{aligned}
2 \xi^{T}(t) N M_{2} \xi(t) & \leq \tau(t) \xi^{T}(t) N\left\{\left(1-2 \alpha \tau_{m}\right) R\right\}^{-1} N^{T} \xi(t) \\
& +\left(1-2 \alpha \tau_{m}\right) \int_{t-\tau(t)}^{t} \dot{x}^{T}(s) R \dot{x}(s) d s
\end{aligned}
$$

the following inequality is obtained:

$$
\begin{gathered}
W^{\alpha}\left(t, x_{t}\right) \leq \xi^{T}(t)\left[\Pi_{1}^{\alpha}+\left(\tau_{m}-\tau(t)\right) \Pi_{2}^{\alpha}\right. \\
\left.+\tau(t) N\left\{\left(1-2 \alpha \tau_{m}\right) R\right\}^{-1} N^{T}\right] \xi(t) .
\end{gathered}
$$

This inequality has to be satisfied for all values of the delay $\tau(t) \in\left[0, \tau_{m}[\right.$. As this equation depends linearly on the delay function, it is necessary and sufficient to ensure the negativity of the matrix at $\tau(t)=0$ and $\tau_{m}$. Applying the Schur complement on the vertice $\tau(t)=\tau_{m}$ leads to the stability conditions of Theorem 4.

The last step of the proof consists in integrating the differential inequality over a sampling interval. This leads to

$$
V\left(t, x_{t}\right)<x^{T}\left(t_{k}\right) \operatorname{Px}\left(t_{k}\right) e^{2 \alpha\left(t-t_{k}\right)} .
$$

The previous expression implies the definition of the exponential stability (11).

The previous theorem can be also extended to the cases of constant sampling period and polytopic systems. For the sake of simplicity, only the case of constant sampling period is provided. The other theorems are not presented here but are straightforward.

Theorem 5: For a given $\alpha>0$, assume that there exist symmetric positive definite matrices $P, R$ and $S \in \mathbb{R}^{n \times n}$, a matrix $U \in \mathbb{R}^{n \times n}$ and a matrix $N \in \mathbb{R}^{2 n \times n}$ that satisfy:

$$
\begin{gathered}
\Psi_{1}^{\alpha}+\tau_{m} \Psi_{2}^{\alpha}<0 \\
{\left[\begin{array}{cc}
\Psi_{1}^{\alpha} & \tau_{m} N \\
* & -\tau_{m}\left(1+2 \alpha \tau_{m}\right) R
\end{array}\right]<0,}
\end{gathered}
$$

where

$$
\begin{gathered}
\Psi_{1}^{\alpha}=\Pi_{1}^{\alpha}-M_{4}^{T} U M_{2}-M_{2}^{T} U^{T} M_{4} \\
\Psi_{2}^{\alpha}=\Pi_{2}^{\alpha}+M_{4}^{T} U M_{3}+M_{3}^{T} U^{T} M_{4} \\
+2 \alpha\left(M_{4}^{T} U M_{2}+M_{2}^{T} U M_{4}\right)
\end{gathered}
$$

and where the matrices $M_{i}$, for $i=1,2,3,4$ are given in Theorems 1 and 2. System (4) is thus exponentially stable with an exponential decay rate $\alpha$ for the constant sampling period $\tau_{m}$.

Remark 2: Theorems 4 and 5 provides sufficient conditions for exponential stability. It allows estimating the 


\begin{tabular}{|c|c|c|}
\hline Time-varying period & - & - \\
\hline Theorems & $\tau_{m}$ & NDV \\
\hline$[3]$ & 0.8696 & $5 n^{2}+2 n$ \\
{$[14]$} & 0.8696 & $7 n^{2}+n$ \\
{$[12]$} & 0.8871 & $16 n^{2}+3 n$ \\
{$[1]$} & 1.009 & $8 n^{2}+4 n$ \\
{$[10]$} & 1.1137 & $3.5 n^{2}+1.5 n$ \\
{$[8]$} & 1.3659 & $0.5\left(n^{2}+n\right)+1$ \\
Th.1 & 1.6894 & $3.5 n^{2}+1.5 n$ \\
\hline Constant period & - & - \\
\hline Theorems & $\tau_{m}$ & NDV \\
\hline$[10]$ & 1.3277 & $5 n^{2}+n$ \\
{$[8]$} & 1.3659 & $0.5\left(n^{2}+n\right)+1$ \\
Th.2 & 1.7198 & $5 n^{2}+n$ \\
\hline Theoretical bound & 1.72 & - \\
\hline
\end{tabular}

TABLE I

MAXIMAL ALLOWABLE SAMPLING PERIOD $\tau_{m}$

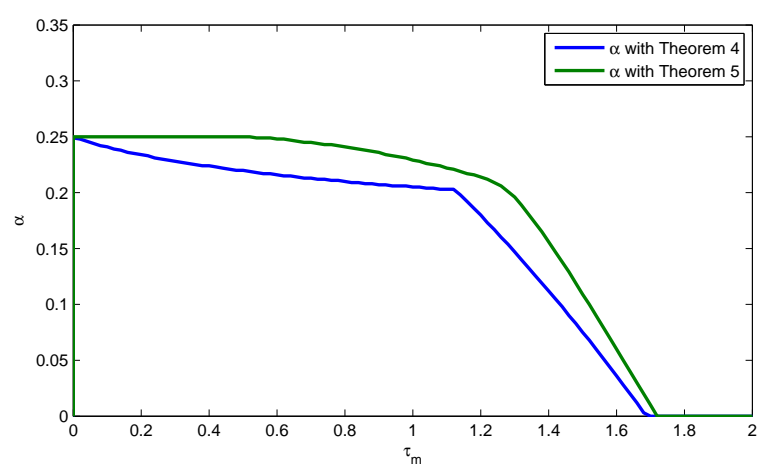

Fig. 2. Relation between the exponential decay rate $\alpha$ and the sampling period $\tau_{m}$ with the cases of constant and time-varying periods for Example1

maximal exponential decay rate $\alpha_{m}$ by solving the following optimization problem:

$$
\alpha_{m}=\max \alpha \text { such that (12) are satisfied }
$$

\section{EXAMPLES}

\section{A. Example 1}

Consider system (4) from [3], [10] with:

$$
A=\left[\begin{array}{cc}
0 & 1 \\
0 & -0.1
\end{array}\right], \quad A_{d}=\left[\begin{array}{cc}
0 & 0 \\
0.375 & -1.15
\end{array}\right] \text {. }
$$

The maximum allowable sampling period from the literature and obtained applying Theorems 1 and 2 are resumed in Table I. It shows both cases of time-varying and constant samplings periods. It can be seen that the results from Theorem 1 and 2 are less conservative than the one from the literature based on continuous-time approaches. Figure 2 shows the relation between the maximal convergence rate $\alpha$ and the sampling period in the constant and time-varying case. It shows that the conditions from Theorem 4 also holds for negative values of $\alpha$.

Remark 3: The theoretical bound given in Table I comes from the computation of the eigenvalues of the matrix which defines system (4) in discrte time. Note that with

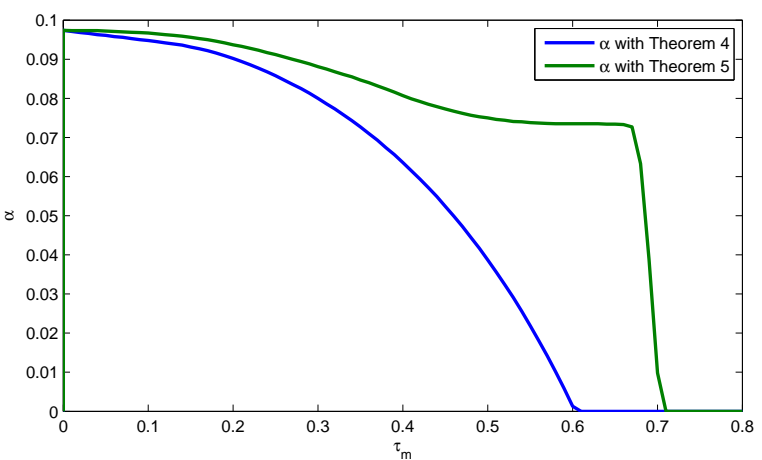

Fig. 3. Relation between the exponential decay rate $\alpha$ and the sampling period $\tau_{m}$ with the cases of constant and time-varying periods for Example2

\section{B. Example 2}

Consider the process model from [3] with:

$$
A=\left[\begin{array}{cc}
1 & 0.5 \\
g_{1} & -1
\end{array}\right], B=\left[\begin{array}{c}
1+g_{2} \\
-1
\end{array}\right] .
$$

where $\left|g_{1}\right| \leq 0.1$, and $\left|g_{2}\right| \leq 0.3$. With the state feedback gain $K=-\left[\begin{array}{ll}2.6884 & 0.6649\end{array}\right]$, in [3] and in [10], it was respectively proven that the system is stable for any sampling interval smaller than $0.35,0.4476$. In this article, Theorem 3 ensures that the system is stable for all samplings whose periods are less than 0.602 . Theorem 3 adapted to the case of constant period ensures that the system is stable all periods less than 0.703 . It is clear that the sufficient stability conditions presented in the paper in hand are less conservative than the ones from the literature. Figure 3 shows the evolution of the convergence rate with respect to the maximum allowable sampling period $\tau_{m}$ for the cases of constant and time-varying periods.

\section{Example 3}

Consider system (4) from [1], [6] with

$$
A=\left[\begin{array}{cc}
-2 & 0 \\
0 & -0.9
\end{array}\right], \quad A_{d}=\left[\begin{array}{cc}
-1 & 0 \\
-1 & -1
\end{array}\right]
$$

The results on asymptotic stability are summarized in Table II. It can be seen that the maximal allowable sampling periods provided by Theorem 1 and 2 are the same as the ones from [10]. In [1], the sufficient conditions for asymptotic stability are satisfied for larger sampling period. This comes from the consideration of particular tools dedicated to time-delay systems that were introduced in this article. Nevertheless, this example shows the limits of the approaches in such case, since the system remains stable, in simulation for some $\tau_{m}$ greater than 3 .

\section{CONCLUSION}

In this article, an analysis of linear invariant and timevarying systems with constant and time-varying sampling periods is provided. Tractable conditions are derived to ensure asymptotic stability and also to obtained an estimate of the convergence rate of the solutions. The examples 


\begin{tabular}{|c|c|c|}
\hline Theorems & $\tau_{m}$ & NDV \\
\hline$[3]$ & 0.8696 & $5 n^{2}+2 n$ \\
{$[14]$} & 0.8696 & $7 n^{2}+n$ \\
{$[12]$} & 0.8871 & $16 n^{2}+3 n$ \\
{$[10]$} & 1.9999 & $3.5 n^{2}+1.5 n$ \\
{$[1]$} & 2.034 & $8 n^{2}+4 n$ \\
Th.1\&2 & 1.9999 & $3.5 n^{2}+1.5 n$ \\
\hline
\end{tabular}

TABLE II

MAXIMAL ALLOWABLE SAMPLING PERIOD $\tau_{m}$ FOR EXAMPLE 3

shows the efficiency of the method and the reduction of the conservatism compared to others results from the literature.

\section{REFERENCES}

[1] Y. Ariba and F. Gouaisbaut, Delay-dependent stability analysis of linear systems with time-varying delay, Proc. of the $46^{\text {th }}$ IEEE Conference on Decision and Control (New Orleans, LA, USA), 2007.

[2] T. Chen and B.A. Francis, Optimal sampled-data control systems, Springer-Verlag, Berlin, Germany, 1995.

[3] E. Fridman, A. Seuret, and J.-P. Richard, Robust sampled-data stabilization of linear systems: An input delay approach, Automatica 40 (2004), no. 8, 1141-1446.

[4] H. Fujioka, Stability analysis of systems with aperiodic sample-andhold devices, Automatica 45 (2009), no. 3, 771-775.

[5] J.P. Hespanha, P. Naghshtabrizi, and Y. Xu, A survey of recent results in networked control systems, Proceedings of the IEEE 95 (2007), no. $1,138-162$.

[6] C. Y. Kao and A. Rantzer, Stability analysis of systems with uncertain time-varying delays, Automatica 43 (2007), 959-970.

[7] X.-G. li, A. Çela, S.-I. Niculescu, and A. Reama, Some remark on the stability of networked control systems with periodic scheduling, Proceedings of the European Control Conference (Budapest, Hongary), 2009.

[8] L. Mirkin, Exponential stability of impulsive systems with application to uncertain sampled-data systems, IEEE Trans. on Automatic Control 52 (2007), no. 6, 1109-1112.

[9] Some remarks on the use of time-varying delay to model sample-and-hold circuits, IEEE Trans. on Automatic Control 52 (2007), no. 6, 1009-1112.

[10] P. Naghshtabrizi, J.P. Hespanha, and A.R. Teel, Exponential stability of impulsive systems with application to uncertain sampled-data systems, Systems and Control Letters 57 (2008), no. 5, 378-385.

[11] S.-I. Niculescu, C.-E. de Souza, L. Dugard, and J.-M. Dion, Robust exponential stability of uncertain systems with time-varying delays, Proceedings of the 33rd IEEE CDC, 1994, p. 431.

[12] D. Yue, Q.-L. Han, and J. Lam, Network-based robust $H^{\infty}$ control of systems with uncertainty, Automatica 41 (2005), no. 6, 640-644.

[13] Stability and stabilization of nonuniform sampling systems, Automatica 44 (2008), no. 12, 3222-3226.

[14] D. Yue, Q.-L. Han, and C. Peng, State feedback controller design for networked control systems, IEEE Trans. on Automatic Control 51 (2004), no. 11, 640-644.

[15] Sandro Zampieri, A survey of recent results in Networked Control Systems, Proc. $17^{\text {th }}$ IFAC World Congress (Seoul, Korea), July 2008.

[16] W. Zhang and M.S. Branicky, Stability of networked control systems with time-varying transmission period, Allerton Conf. Communication, Control, and Computing, October 2001.

[17] W. Zhang, M.S. Branicky, and S.M. Phillips, Stability of networked control systems, IEEE Control Systems Magazine (2001), no. 21. 